\title{
Analysis of the Conduction-Radiation Problem in Absorbing, Emitting, Non-Gray Planar Media Using an Exact Method
}

Travis J. Moore

Brigham Young University - Provo, travisjmoore@gmai.com

Matthew R. Jones

Brigham Young University - Provo, mrjones@byu.edu

Follow this and additional works at: https://scholarsarchive.byu.edu/facpub

Part of the Engineering Commons

\section{Original Publication Citation}

Travis J. Moore, Matthew R. Jones, Analysis of the conduction-radiation problem in absorbing, emitting, non-gray planar media using an exact method, International Journal of Heat and Mass Transfer, Volume 73, 2014, Pages 804-809, ISSN 0017-9310, https://doi.org/10.1016/ j.ijheatmasstransfer.2014.02.029. (http://www.sciencedirect.com/science/article/pii/ S001793101400146X)

\section{BYU ScholarsArchive Citation}

Moore, Travis J. and Jones, Matthew R., "Analysis of the Conduction-Radiation Problem in Absorbing, Emitting, Non-Gray Planar Media Using an Exact Method" (2014). Faculty Publications. 3157.

https://scholarsarchive.byu.edu/facpub/3157 
Q1 Travis J. Moore*, Matthew R. Jones

Department of Mechanical Engineering, Brigham Young University, 435 Crabtree Building, Provo, UT 84602, USA

\begin{abstract}
A B S T R A C T
Systems in which both conduction and radiation are the dominant modes of heat transfer are important in many engineering applications and various numerical methods exist to analyze such systems. An exact solution to the conduction-radiation problem in a one-dimensional, planar, absorbing, emitting, nongray medium is presented. The method uses an integrating factor to solve the radiative transfer equation and variation of parameters is used to solve the energy equation. The model is verified by comparing the temperature profiles calculated from this work to those found using numerical methods for both gray and
\end{abstract} non-gray cases.

(c) 2014 Elsevier Ltd. All rights reserved.

\section{A R T I C L E I N F O}

\section{Article history:}

Received 22 October 2013

Received in revised form 10 February 2014

Accepted 12 February 2014

Available online $\mathrm{xxxx}$

\section{Keywords:}

Conduction-radiation

Plane parallel

Non-gray

Participating medium

Exact methods

\section{Introduction}

Systems in which both conduction and radiation are the dominant modes of heat transfer can be found in many practical engineering applications. These include fiber and foam insulations, the manufacture of glass, the study and design of furnaces and internal-combustion engines, filler and cover for windows and solar collectors, thermal barrier coatings, and many others. The wide variety of applications has resulted in several numerical and experimental studies of methods for analyzing systems with simultaneous conduction and radiation.

The analysis of such systems is inherently difficult because of the integro-differential nature of the radiative transfer equation (RTE) [1]. The pioneering theoretical analysis for this problem was presented by Viskanta and Grosh in 1962 in which the temperature profile of a one-dimensional, gray medium bounded on both sides by opaque surfaces was obtained by transforming the governing integro-differential equation into a non-linear integral equation that was solved iteratively [2]. The same authors investigated the effects of different emittances of the bounding surfaces on the heat transfer in the gray medium [3]. The results of these analyzes are often used as benchmark solutions to which other methods are compared.

\footnotetext{
* Corresponding author. Tel.: +1 8017098754.

E-mail addresses: travisjmoore@gmail.com (T.J. Moore), mrjones@byu.edu (M.R. Jones).
}

More recent approaches to solving the combined conductionradiation problem include the use of the finite element method $[4,5]$, the finite difference method [6,7], the finite volume method $[8,9]$, and the lattice Boltzmann method [10-12]. The radiation portion of the problem has been solved using the discrete ordinates method [11], the discrete transfer method [1,12], the method of spherical harmonics $[1,13]$, the Monte Carlo method [14], and the finite volume method $[10,15]$. Each of these methods has advantages and disadvantages [16].

Most of the published solutions deal with heat transfer in gray media [17]. Fewer investigations have been conducted for the case of conduction-radiation heat transfer in non-gray media. Heinemann et al. used theoretical and experimental methods to analyze conduction-radiation heat transfer in non-gray silica aerogels [18] while Manohar et al. investigated conduction-radiation heat transfer in non-gray plastics [19]. More recently, Marques et al. developed a computationally efficient numerical model based on finite strip theory to determine the temperature profile and heat flux in absorbing, emitting, non-scattering, non-gray media [17].

The solution presented here resulted from an investigation into the temperature gradient in thermal barrier coatings (TBCs). Thermal barrier coatings allow for increased inlet temperatures in power generation and aerospace turbines, thereby increasing efficiency and reducing air cooling requirements. Knowledge of the temperature profile in a thermal barrier coating is critical for evaluating the TBC performance and monitoring its health, as well as for accurate simulation and modeling. Another current application of the solution presented in this paper is determination of the tem- 


$\begin{array}{ll}\text { Nomenclature } \\ E & \text { emissive power } \\ H & \text { terms in summation of temperature profile } \\ I & \text { radiative intensity } \\ k & \text { thermal conductivity } \\ L & \text { length of medium } \\ N & \text { conduction-to-radiation parameter } \\ P & \text { function of } z, \lambda \text {, and } \mu \\ Q & \text { function of } z, \lambda \text {, and } \mu \\ q & \text { heat flux } \\ R & \text { function of } \lambda \text { and } \mu \\ \hat{S} & \text { direction vector } \\ T & \text { temperature } \\ z & \text { Cartesian coordinate } \\ \text { Greek symbols } \\ \theta & \text { emittance of boundary } \\ \theta & \text { polar angle } \\ \kappa & \text { absorption coefficient } \\ \lambda & \text { wavelength }\end{array}$

perature profile in blackbody optical fiber pyrometers [20-22]. A blackbody optical fiber pyrometer consists of an optical fiber whose sensing tip is coated with a highly conductive, opaque material. The blackbody radiation emitted by the tip of the fiber is transmitted along the optical fiber to a detector. Correct interpretation of these emission measurements requires a solution to the coupled radiation-conduction problem. The use of blackbody optical fiber pyrometers in oxy-combustion flames is currently being investigated. Oxy-combustion is a promising technology being considered for carbon capture and sequestration due to the fact it has the potential to produce very low levels of emissions for all of the major pollutants of coal and natural gas. An understanding of the gas temperatures is critical to oxy-combustion development. Blackbody optical fiber pyrometry has the potential to solve all of the major problems associated with measuring temperatures in large-scale, particle-laden combustion systems that are required for the development of oxy-combustion.

Previous efforts to solve combined conduction and radiation heat transfer problems have relied on numerical methods to solve the governing equations. The present work outlines an exact solution to the equations governing the simultaneous conduction and radiation heat transfer in a one-dimensional, plane parallel, absorbing, emitting, non-scattering, non-gray medium surrounded by diffuse, opaque surfaces. The particular solution to the governing differential equation is obtained using the method of variation of parameters while the spectral intensities required to calculate the total radiative heat flux are found by solving the RTE using an integrating factor. This approach results in an integral equation that is solved for the temperature profile. The temperature profile is obtained using iterative, numerical integration, and a closedform solution is not obtained. However, since numerical integration can be performed to an arbitrary degree of precision, the solution is exact. The model is verified by comparing the results for various cases to those calculated using different numerical methods and to CFD simulations performed using commercial software [23], which employs the discrete ordinates method to model the radiative heat transfer.

\section{Problem formulation}

A one-dimensional, plane-parallel, homogeneous, isotropic, non-gray, participating medium bounded by two surfaces is shown $\mu \quad$ cosine of intensity direction

$\rho \quad$ reflectance of boundary

$\sigma \quad$ Stefan-Boltzmann constant

$\phi \quad$ azimuthal angle

$\Omega \quad$ solid angle

Subscripts

0 at left boundary

b blackbody

$L \quad$ at right boundary

$i, m, n \quad$ indices

$R \quad$ radiative

$s \quad$ in the $\hat{s}$ direction

$\lambda \quad$ spectrally dependent

Superscripts

$+\quad$ forward direction

- backward direction

integration coordinate in Fig. 1. The medium is absorbing, emitting, and non-scattering and the bounding surfaces are opaque and diffuse. The temperature of the boundaries are denoted by $T_{0}$ and $T_{L}$ at $z=0$ and $z=L$, respectively. The material properties of both the participating medium and the boundaries are independent of temperature. Heat transfer in the medium occurs by both conduction and radiation. The energy equation for simultaneous conduction and radiation for a one-dimensional, planar medium at steady state with uniform properties reduces to [1]

$\frac{d^{2} T}{d z^{2}}=\frac{1}{k} \frac{d q_{R}}{d z}$

Eq. (1) is subject to the following boundary conditions.

$T(0)=T_{0}$

$T(L)=T_{L}$

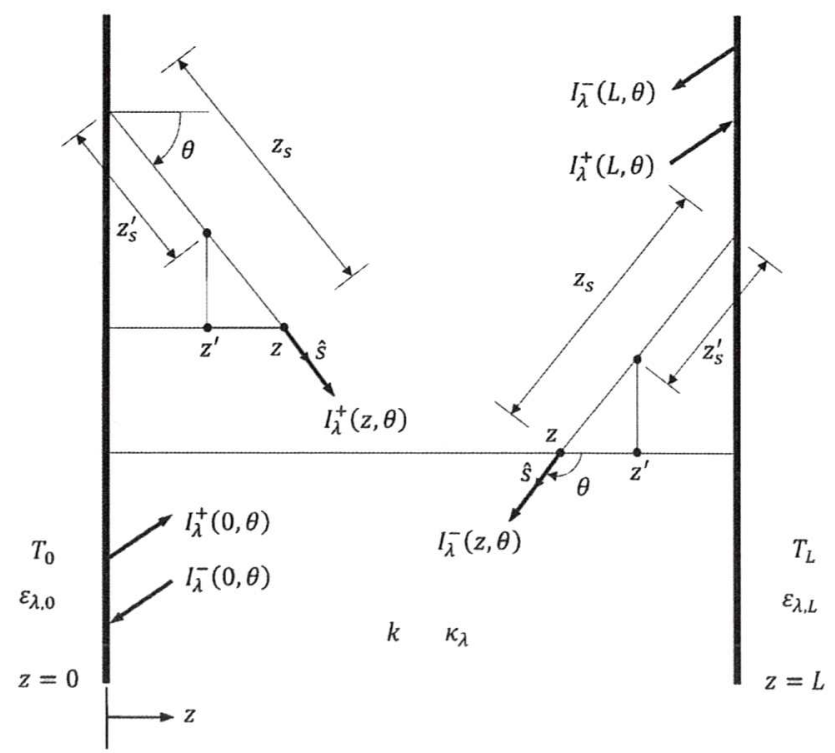

Fig. 1. Absorbing-emitting medium between two diffuse boundaries. Coordinates used in the theoretical analysis are shown. 
Solving for the temperature profile in the medium requires determination of the total radiative heat flux, $q_{R}$. Assuming azimuthal symmetry and applying the variable transformation $\mu=\cos \theta$, the total radiative heat flux may be expressed as [1]

$$
\begin{aligned}
q_{R} & =2 \pi \int_{0}^{\infty} \int_{-1}^{+1} I(z, \mu) \mu d \mu d \lambda \\
& =2 \pi \int_{0}^{\infty}\left[\int_{0}^{1} I_{\lambda}^{+}(z, \mu) \mu d \mu-\int_{0}^{1} I_{\dot{\lambda}}^{-}(z,-\mu) \mu d \mu\right] d \lambda
\end{aligned}
$$

The RTE is used to calculate $I_{\lambda}^{+}(z, \mu)$ and $I_{\lambda}^{-}(z,-\mu)$. For a non-scattering medium and looking in the $z_{s}$ direction from the left boundary, the RTE reduces to [1]

$\frac{d I_{\lambda}^{+}}{d z_{s}}=\kappa_{\lambda} I_{b, \lambda}\left(z_{s}\right)-\kappa_{\lambda} I_{\lambda}^{+}$

The formal solution of Eq. (5) along a line of sight, which is obtained by employing an integrating factor, is [1]

$I_{\lambda}^{+}\left(z_{s}\right)=I_{\lambda}^{+}(0) e^{-\kappa_{\lambda} z_{s}}+\kappa_{\lambda} \int_{0}^{z_{s}} I_{b, \lambda}\left(T\left(z_{s}^{\prime}\right)\right) e^{-\kappa_{\lambda}\left(z_{s}-z_{s}^{\prime}\right)} d z_{s}^{\prime}$

Beginning at an arbitrary point on the right boundary and repeating this solution process gives the needed expression for $I_{\lambda}^{-}\left(z_{s}\right)$.

$$
\begin{aligned}
I_{\lambda}^{-}\left(z_{s}\right)= & I_{\lambda}^{-}(L / \cos \theta) e^{\kappa_{\lambda}\left(L / \cos \theta-z_{s}\right)}-\kappa_{\lambda} \\
& \times \int_{z_{s}}^{L / \cos \theta} I_{b, \lambda}\left(T\left(z_{s}^{\prime}\right)\right) e^{\kappa_{\lambda}\left(z_{s}^{\prime}-z_{s}\right)} d z_{s}^{\prime}
\end{aligned}
$$

Eqs. (6) and (7) can be rewritten using the variable transformation $z_{s}=z / \cos \theta$ and in terms of the direction $\operatorname{cosine} \mu=\cos \theta$ as follows.

$I_{\lambda}^{+}(z, \mu)=I_{\dot{\lambda}}^{+}(0, \mu) e^{-\kappa_{;} z / \mu}+\frac{\kappa_{\lambda}}{\mu} \int_{0}^{z} I_{b, \lambda}\left(T\left(z^{\prime}\right)\right) e^{-\kappa_{\lambda}\left(z-z^{\prime}\right) / \mu} d z^{\prime}$

$$
I_{\lambda}^{-}(z,-\mu)=I_{\lambda}^{-}(L,-\mu) e^{-\kappa_{\lambda}(L-z) / \mu}+\frac{\kappa_{\lambda}}{\mu} \int_{z}^{L} I_{b, \lambda}\left(T\left(z^{\prime}\right)\right) e^{-\kappa_{\lambda}\left(z^{\prime}-z\right) / \mu} d z^{\prime}
$$

The boundary conditions, $I_{\lambda}^{+}(0, \mu)$ and $I_{\lambda}^{-}(L,-\mu)$, must now be specified. $I_{\lambda}^{+}(0, \mu)$ represents the intensity coming from the left boundary, which is due to both diffuse emission and reflection and is represented by Eq. (10).

$I_{\lambda}^{+}(0, \mu)=\varepsilon_{\lambda, 0} I_{b, \lambda}(T(0))+\rho_{\dot{\lambda}, 0} I_{\dot{\lambda}}^{-}(0,-\mu)$

The first term on the right side of Eq. (10) represents the diffuse emission from the wall while the second term is the diffuse reflection of the incident intensity on the wall where $\rho_{\lambda, 0}$ is the spectral, directional-hemispherical reflectance. The incident intensity, $I_{i}^{-}(0,-\mu)$, is a result of the emission and reflection from the opposite wall as well as the emission from the medium itself and is therefore dependent on direction. $I_{\lambda}^{-}(0,-\mu)$ is found by letting $z=0$ in Eq. (9). Similarly, $I_{\lambda}^{-}(L,-\mu)$ represents the intensity emitted and reflected by the right surface.

$I_{\lambda}^{-}(L,-\mu)=\varepsilon_{\lambda, L} I_{b, \lambda}(T(L))+\rho_{\lambda, L} I_{\lambda}^{+}(L, \mu)$

The intensity incident on the left boundary, $I_{\lambda}^{+}(L, \mu)$, is found by letting $z=L$ in Eq. (8).

\section{Solution}

Eqs. (10) and (11) are used in Eqs. (8) and (9) to determine expressions for $I_{\lambda}^{+}(z, \mu)$ and $I_{\lambda}^{-}(z,-\mu)$. These expressions are substituted into Eq. (4), resulting in an expression for the total radiative heat flux. This, in turn, is substituted into Eq. (1) and Eqs (1)-(3) are solved using the method of variation of parameters. Although primarily used to solve linear differential equations, the method of variation of parameters has been used to solve nonlinear differential equations, particularly those in which the nonhomogeneity is a function of the dependent variable [24-27]. The details of this solution are provided by Moore [28]. The result, shown in Eq. (12), is an expression for the temperature profile in a one-dimensional, plane-parallel, non-gray, absorbing, emitting, and non-scattering medium bounded by two opaque surfaces.

$T(z)=\left(\frac{T_{L}}{L}-\frac{T_{0}}{L}\right) z+T_{0}+\frac{2}{k} \sum_{i=1}^{12} \int_{0}^{\infty} \int_{0}^{1} H_{i}(z, \lambda, \mu) d \mu d \lambda$

The terms in the summation are shown in Table 1 . In the terms in Table 1 ,

$P(z, \lambda, \mu)=\frac{\mu}{\left(1-\rho_{\dot{\lambda}, 0} \rho_{\lambda, L} e^{-2 \kappa_{\lambda} L / \mu}\right)}\left(\frac{z}{L}\left(e^{-\kappa_{\lambda} L / \mu}-1\right)+\left(1-e^{-\kappa_{j} z / \mu}\right)\right)$

$Q(z, \lambda, \mu)=\frac{\mu}{\left(1-\rho_{\dot{\lambda}, 0} \rho_{\lambda, L} e^{-2 \kappa_{\lambda} L / \mu}\right)}\left(\frac{z}{L}\left(1-e^{-\kappa_{i} L / \mu}\right)+e^{-\kappa_{\lambda} L / \mu}\left(1-e^{\kappa_{\lambda} z / \mu}\right)\right)$

\section{Evaluation}

The solution for the temperature profile given in Eq. (12) is not a closed-form solution, so evaluation of the temperature profile requires an iterative approach involving the numerical integration of the $H_{i}(z, \lambda, \mu)$ terms over direction and wavelength. Although numerical methods are used in the iterative approach, numerical integration can be performed to an arbitrary degree of precision, so the solution is exact.

The iterative solution begins with an initial guess of the temperature profile. In this study, the linear temperature profile that results from neglecting the radiative transfer is used as the initial guess. With this initial guess, the spectral emissive power is calculated, and the temperature profile is updated using Eq. (12). This updated temperature profile is then used to recalculate the spectral emissive power as needed and the process is repeated until convergence is obtained. Convergence is considered achieved when the Euclidean norm of the difference between the calculated temperature profile of the current iteration and that of the previous iteration reaches a specified tolerance. In cases where the radiation heat transfer is dominant, under-relaxation is required to obtain convergence.

When numerically evaluating the integrals in Eq. (12), both the spatial dimension, $z$, and the direction cosine, $\mu$, are discretized and each integral is expressed as a summation. For example, the eleventh term in the summation is approximated as

$$
\begin{aligned}
H_{11} & =\left(1-\frac{z}{L}\right) \int_{0}^{\infty} \int_{0}^{1} \int_{0}^{L} E_{b . \lambda}\left(T\left(z^{\prime}\right)\right) e^{-\kappa_{k} z^{\prime} / \mu} \mu d z^{\prime} d \mu d \lambda \\
& \approx\left(1-\frac{z}{L}\right) \int_{0}^{\infty} \sum_{m} \sum_{n} E_{b . \lambda}\left(T\left(z_{n}\right)\right) e^{-\kappa_{\lambda} z_{n} / \mu_{m}} \mu_{m} \Delta z \Delta \mu d \lambda
\end{aligned}
$$

Again, although numerical methods are used to evaluate the integrals, the degree of precision is based on the step sizes $\Delta z$ and $\Delta \mu$ and, therefore, the solution can still be considered exact. For gray media, the Stefan-Boltzmann Law [1] may be used to evaluate the integral of the spectral blackbody emissive power over all wavelengths. For non-gray media, the spectrally varying properties are discretized into bands and assumed to be constant over each band. The spectral emissive power in each band is calculated using a curve fit to the fractional blackbody emissive power function $[1,29]$. 
Table 1

Terms of the summation in Eq. (12).

\begin{tabular}{|c|c|c|c|}
\hline$H_{1}$ & $\frac{\varepsilon_{, 0}, 0}{\kappa_{i}} E_{b, \lambda}(T(0)) P(z, \lambda, \mu) \mu$ & $\mathrm{H}_{7}$ & $\frac{\varepsilon_{\mu \lambda}}{\kappa_{\lambda}} E_{b, \lambda}(T(L)) Q(z, \lambda, \mu) \mu$ \\
\hline $\mathrm{H}_{2}$ & $\frac{\rho_{i, 0} \varepsilon_{\lambda L}}{\kappa_{\lambda}} E_{b, \lambda}(T(L)) e^{-\kappa_{\lambda} L / \mu} P(z, \lambda, \mu) \mu$ & $H_{8}$ & $\frac{\rho_{\lambda \ell_{2, O}}}{\kappa_{\lambda}} E_{b, \lambda}(T(0)) e^{-\kappa_{\lambda} L / \mu} Q(z, \lambda, \mu) \mu$ \\
\hline $\mathrm{H}_{3}$ & $\rho_{\lambda, 0} \rho_{\lambda, L} P(z, \lambda, \mu) \int_{0}^{L} E_{b, \lambda}\left(T\left(z^{\prime}\right)\right) e^{-\kappa_{i}\left(2 L-z^{\prime}\right) / \mu} d z^{\prime}$ & $H_{9}$ & $\rho_{\lambda, L} Q(z, \lambda, \mu) \int_{0}^{L} E_{b, \lambda}\left(T\left(z^{\prime}\right)\right) e^{-\kappa_{\lambda}\left(L-z^{\prime}\right) / \mu} d z^{\prime}$ \\
\hline $\mathrm{H}_{4}$ & $\rho_{\lambda, 0} P(z, \lambda, \mu) \int_{0}^{L} E_{b, \lambda}\left(T\left(z^{\prime}\right)\right) e^{-\kappa_{\lambda} z^{\prime} / \mu} d z^{\prime}$ & $H_{10}$ & $\rho_{\lambda, 0} \rho_{i, L} Q(z, \lambda, \mu) \int_{0}^{L} E_{b, \lambda}\left(T\left(z^{\prime}\right)\right) e^{-k_{i}\left(L+z^{\prime}\right) / \mu} d z^{\prime}$ \\
\hline $\mathrm{H}_{5}$ & $\frac{z}{L} \mu \int_{0}^{L} E_{b, \lambda}\left(T\left(z^{\prime}\right)\right) e^{-\kappa_{i}\left(L-z^{\prime}\right) / \mu} d z^{\prime}$ & $H_{11}$ & $\left(1-\frac{z}{t}\right) \mu \int_{0}^{L} E_{b, \lambda}\left(T\left(z^{\prime}\right)\right) e^{-\kappa_{\lambda} z^{\prime} / \mu} d z^{\prime}$ \\
\hline$H_{6}$ & $-\mu \int_{0}^{z} E_{b, \lambda}\left(T\left(z^{\prime}\right)\right) e^{-\kappa_{\lambda}\left(z-z^{\prime}\right) / \mu} d z^{\prime}$ & $H_{12}$ & $-\mu \int_{z}^{L} E_{b, \lambda}\left(T\left(z^{\prime}\right)\right) e^{-\kappa_{i}\left(z^{\prime}-z\right) / \mu} d z^{\prime}$ \\
\hline
\end{tabular}

\section{Results and discussion}

The evaluation methodology discussed in Section 4 was implemented as a program in MATLAB. The accuracy of the method is verified by comparing the results for various cases to those calculated using different numerical methods and to CFD simulations in which the discrete-ordinates method is used to model the radiative heat transfer [23]. The conduction-to-radiation parameter is defined as

$N=\frac{k \kappa}{4 \sigma T_{0}^{3}}$

For optically thick slabs, this parameter provides a good estimate for the relative importance of conduction heat transfer relative to radiation heat transfer [1]. The simplest case is a gray material with $\kappa=1 \mathrm{~m}^{-1}$ surrounded by black surfaces $\left(\varepsilon_{0}=\varepsilon_{L}=1\right)$ with $T_{L}=0.5 T_{0}$. For this simple case, the non-dimensional temperature profiles calculated using Eq. (12) are compared to those calculated using a finite difference method [1], the collapsed dimension method implemented by Talukdar and Mishra [30], and the discrete ordinates method [23]. Fig. 2 shows these comparisons for different values of $N$. The average difference between the results of the present method and the numerical methods is $0.89 \%$. The number of iterations required by the collapsed dimension method for $N=0.1$ and 0.01 was 80 and 120 [26], respectively while that of the method presented here was 7 and 37, respectively. The computational time required for the present method was, on average, about 3.5 times faster than that of the finite element method but was, on average, about three times slower than that of the CFD simulations.

A somewhat more complicated case is a gray medium surrounded by non-black surfaces. Fig. 3 compares the temperature

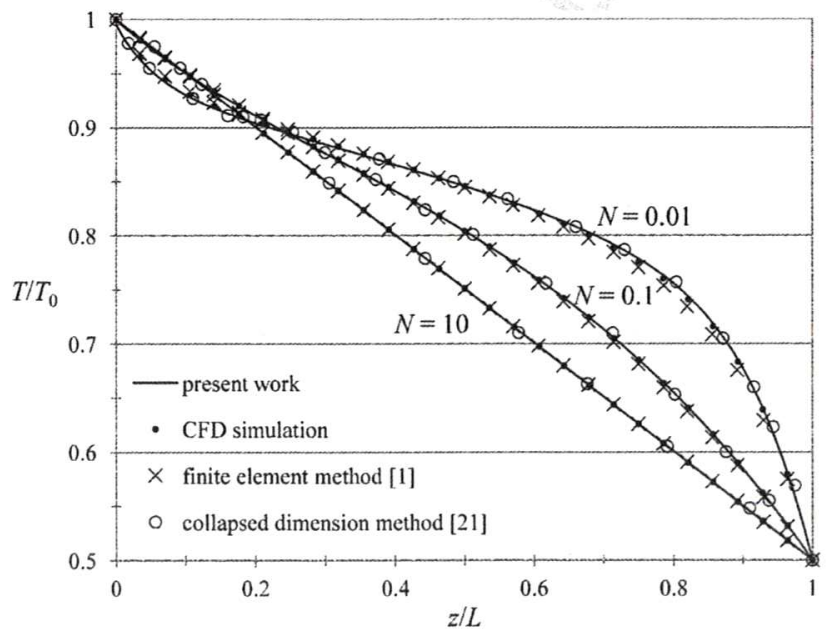

Fig. 2. Comparison of present method to numerical methods for case of gray medium $(k=1)$ with black boundaries for various conduction-to-radiation parameters.

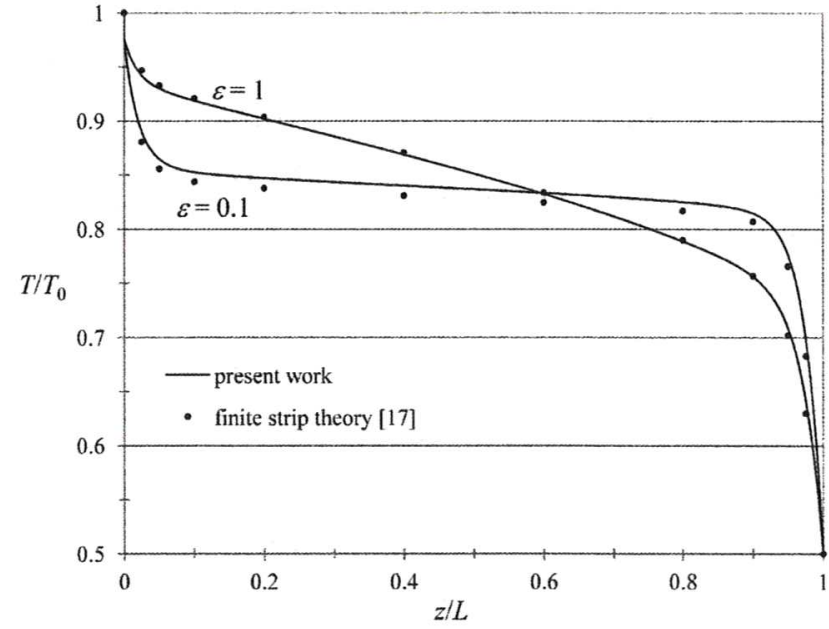

Fig. 3. Comparison of present method to finite strip method [17] for case of gray medium $(k=1)$ with $\varepsilon_{0}=\varepsilon_{L}=1$ and $\varepsilon_{0}=\varepsilon_{\mathrm{L}}=0.1$ for $N=0.001$.

profiles found using the present method to those found using finite strip theory [17] for a gray medium with $\kappa=1 \mathrm{~m}^{-1}$ and $N=0.001$ with boundary emittances of $\varepsilon_{0}=\varepsilon_{L}=1$ and $\varepsilon_{0}=\varepsilon_{L}=0.1$. Figs. 4 and 5 compare the results from this work to those found from CFD simulations for cases in which the boundary emittances are different. Fig. 4 shows the case of a gray medium with $\kappa=0.1 \mathrm{~m}^{-1}$ and boundary emittances of $\varepsilon_{0}=0.75$ and $\varepsilon_{L}=0.1$ while Fig. 5 shows the case of a gray medium with $\kappa=1 \mathrm{~m}^{-1}$ and boundary emittances of $\varepsilon_{0}=0.2$ and $\varepsilon_{L}=0.9$. The bounding surfaces are assumed to be diffuse, so $\rho=1-\varepsilon$ in each of these cases. The average differ-

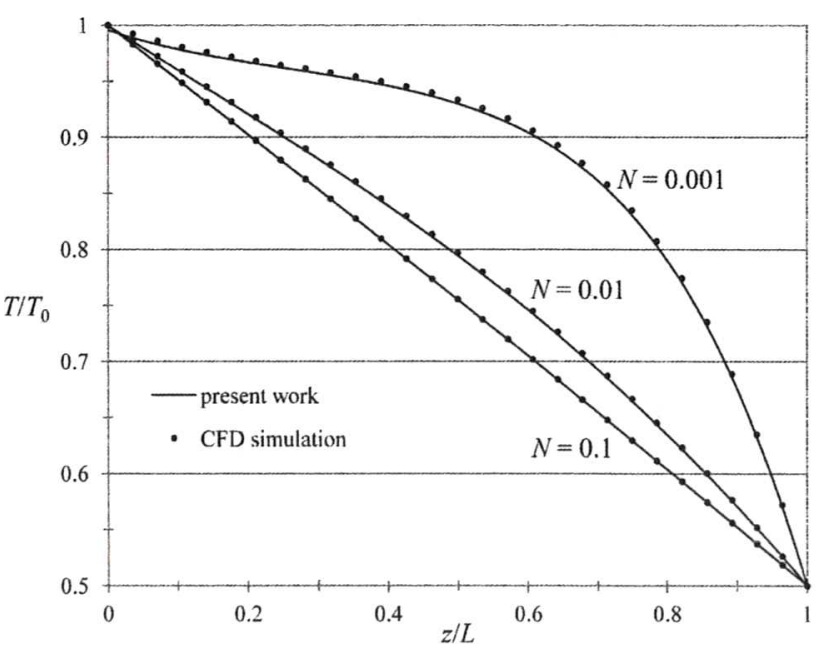

Fig. 4. Comparison of present method to CFD solutions for case of gray medium $(k=0.1)$ with $\varepsilon_{0}=0.75$ and $\varepsilon_{\mathrm{L}}=0.1$ for various conduction-to-radiation parameters. 


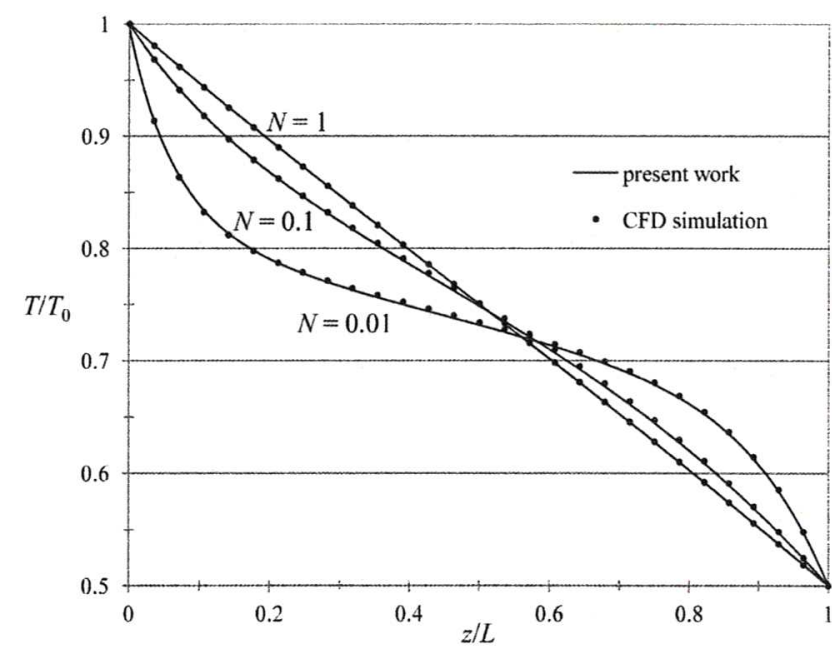

Fig. 5. Comparison of present method to CFD solutions for case of gray medium $(k=1)$ with $\varepsilon_{0}=0.2$ and $\varepsilon_{\mathrm{L}}=0.9$ for various conduction-to-radiation parameters.

Table 2

Spectral properties for two non-gray cases.

\begin{tabular}{llll}
\hline Case $1:$ & $T_{0}=800 \mathrm{~K}, T_{L}=400 \mathrm{~K}$ & & \\
Band & $0-10 \mu \mathrm{m}$ & $10-20 \mu \mathrm{m}$ & $20-\infty \mu \mathrm{m}$ \\
$\varepsilon_{\lambda, 0}$ & 0.25 & 0.5 & 0.75 \\
$\varepsilon_{\lambda, L}$ & 0.3 & 0.85 & 1 \\
$\kappa_{\lambda}$ & 10 & 1 & 0.1 \\
Case 2: $T_{0}=1400 \mathrm{~K}, T_{L}=900 \mathrm{~K}$ & & \\
Band & $0-2 \mu \mathrm{m}$ & $2-7 \mu \mathrm{m}$ & $7-\infty \mu \mathrm{m}$ \\
$\varepsilon_{\lambda, 0}$ & 0.5 & 0.1 & 0.3 \\
$\varepsilon_{\lambda, L}$ & 0.65 & 0.9 & 0.8 \\
$\kappa_{\lambda}$ & 5 & 0.2 & 2 \\
\hline
\end{tabular}

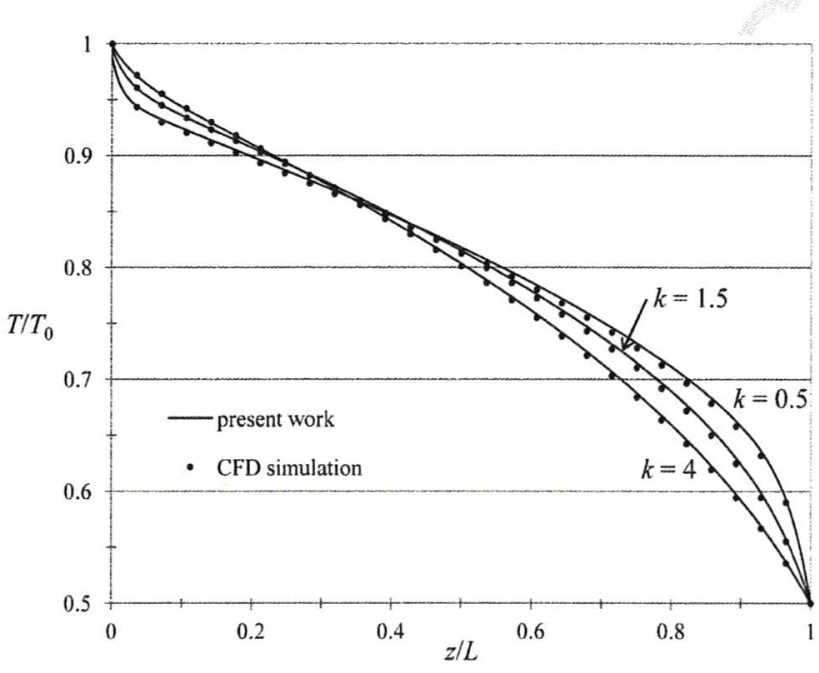

Fig. 6. Comparison of present method to CFD solutions non-gray for various thermal conductivities. Properties correspond to Case 1 in Table 2.

ence between the results of the present method and the numerical methods is $1.2 \%$. The computational time required for the present method was, on average, about eight times greater than that of the CFD simulations.

Finally, the most detailed case is that of a non-gray medium bounded by spectrally selective surfaces. Two scenarios are considered. The spectrally dependent properties of the medium and boundaries are divided into three bands over each of which the properties are assumed to be constant. Table 2 shows the values

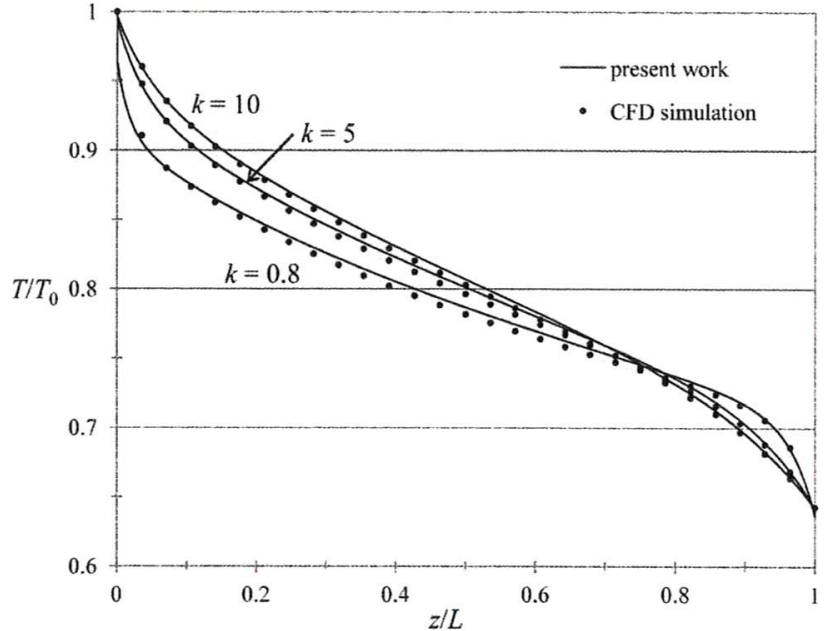

Fig. 7. Comparison of present method to CFD solutions non-gray for various thermal conductivities. Properties correspond to Case 2 in Table 2.

of these spectrally dependent properties for the two cases and the results of these cases are compared to CFD simulations and shown in Figs. 6 and 7 for various values of the thermal conductivity. Again, the bounding surfaces are assumed to be diffuse, so $\rho_{i}=1-\varepsilon_{\lambda}$. The average difference between the results of the present method and the CFD solutions is $1.67 \%$. The computational time required for the present method was, on average, about 55 times greater than that of the CFD simulations.

As can be seen from the figures above, there is excellent agreement between the model presented in this work and other methods for determining the temperature profile in a gray or non-gray one-dimensional, plane-parallel medium surrounded by two gray or non-gray diffuse, opaque surfaces. The average difference between the results of the present method and the numerical methods tested was $1.25 \%$. The time required for the present model to reach convergence was often greater than that required for the other methods, especially the CFD simulations. This was most apparent in the non-gray cases. This was, in part, due to the need to use an under relaxation factor that decreased as the conduction-to-radiation parameter decreased and as the thermal conductivity of the medium decreased. The time required for convergence may be improved by using a dynamic under relaxation factor. Additionally, a large number of points were required in the discretization of the spatial coordinate in order to fully capture the effects of the radiative heat transfer at the left boundary, which significantly increased the time required to achieve convergence. The use of a dynamic under relaxation factor and non-uniform spatial discretization would significantly improve the computational efficiency of the exact solution. The model presented here is, in theory, more accurate than the numerical methods and may be used as a benchmark solution for validation of other numerical methods.

\section{Conclusions}

An exact, analytical method of determining the temperature profile in a one-dimensional, planar, absorbing, emitting, non-gray medium in which both radiation and conduction heat transfer are significant has been presented. The method utilizes an integrating factor to solve the Radiative Transfer Equation to determine the spectral intensities required in the total radiative heat flux. The method of variation of parameters is employed to solve the energy equation in which the divergence of the radiative heat flux is the source of thermal energy. Comparison of results obtained using 
the proposed method with results obtained using various numerical techniques has verified the method. Cases with gray and nongray media and gray and non-gray boundaries were considered. Since the proposed method is exact, it may be used to obtain benchmark solutions.

\section{References}

[1] M.F. Modest, Radiative Heat Transfer, second ed., Academic Press, 2003.

[2] R. Viskanta, R.J. Grosh, Heat transfer by simultaneous conduction and radiation in an absorbing medium, J. Heat Transfer 84 (1) (1962) 63-72.

[3] R. Viskanta, R.J. Grosh, Effect of surface emissivity on heat transfer by simultaneous conduction and radiation, Int. J. Heat Mass Transfer 5 (8) (1962) 729-734.

[4] S.M.H. Sarvari, J.R. Howell, S.H. Mansouri, Inverse boundary design conduction-radiation problem in irregular two-dimensional domains, Numer. Heat Transfer, Part B 44 (3) (2003) 209-224.

[5] L.M. Ruan, M. Xie, H. Qi, W. An, H.P. Tan, Development of a finite element model for coupled radiative and conductive heat transfer in participating media, J. Quant. Spectrosc. Radiat. Transfer 102 (2) (2006) 190-202.

[6] F. Asllanaj, A. Milandri, G. Jeandel, J.R. Roche, A finite difference solution of non-linear systems of radiative-conductive heat transfer equations, Int. J. Numer. Meth. Eng. 54 (11) (2002) 1649-1668.

[7] B. Safavisohi, E. Sharbati, C. Aghanajafi, R.K. Firoozabadi, Finite difference solution for radiative-conductive heat transfer of a semitransparent polycarbonate layer, J. Appl. Polym. Sci. 112 (6) (2009) 3313-3321.

[8] S.V. Patankar, Numerical Heat Transfer and Fluid Flow, first ed., Hemisphere Publishing Corp, 1980.

[9] K. Birgelis, U. Raitums, Convergence of the finite volume method for a conductive-radiative heat transfer problem, Math. Model. Anal. 18 (2) (2013) 274-288.

[10] S.C. Mishra, H.K. Roy, Solving transient conduction-radiation problems using the lattice Boltzmann method and the finite volume method, J. Comput. Phys. 223 (1) (2007) 89-107.

[11] B. Mondal, S.C. Mishra, Application of the lattice Boltzmann method and discrete ordinate method for solving transient conduction and radiation heat transfer problems, Numer. Heat Transfer, A 52 (8) (2007) 757-775.

[12] S.C. Mishra, A. Lankadasu, Transient conduction-radiation heat transfer in participating media using the lattice Boltzmann method and the discrete transfer method, Numer. Heat Transfer, A 47 (9) (2005) 935-954.

[13] J.H. Tsai, Double spherical-harmonics approximation applied to combined conduction-radiation in a planar medium, Int. Commun. Heat Mass Transfer 18 (5) (1991) 741-756.
[14] A.E. Kovtanyuk, N.D. Botkin, K.H. Hoffmann, Numerical simulations of coupled radiative-conductive heat transfer model using a modified Monte Carlo method, Int. J. Heat Mass Transfer 55 (4) (2012) 649-654.

[15] J.C. Chai, H.S. Lee, S.V. Patankar, Finite volume method for radiation heat transfer, J. Thermophys. Heat Transfer 8 (3) (1994) 419-425.

[16] S.C. Mishra, M. Prasad, Radiative heat transfer in participating media - a review, Sadhana 23 (2) (1998) 213-232.

[17] S.P.C. Marques, E.J. Barbero, J.S.R. Murillo, Analysis of conduction-radiation problem in absorbing and emitting nongray materials, Int. J. Numer. Meth. Heat Fluid Flow 19 (2) (2009) 165-181.

[18] U. Heinemann, R. Caps, J. Fricke, Radiation-conduction interaction: an investigation on silica aerogels, Int. J. Heat Mass Transfer 39 (10) (1996) 2115-2130.

[19] S.S. Manohar, A.K. Kulkarni, S.T. Thynell, In-depth absorption of externally incident radiation in nongray media, J. Heat Transfer 117 (1) (1995) 146-151.

[20] D.G. Barker, M.R. Jones, Inversion of spectral emission measurements to reconstruct the temperature profile along a blackbody optical fiber thermometer, Inverse Prob. Eng. 11 (6) (2003) 495-513.

[21] D.G. Barker, M.R. Jones, Temperature measurements using a high-temperature blackbody optical fiber thermometer, J. Heat Transfer 125 (3) (2003) 471-477.

[22] M.R. Jones, D.G. Barker, Use of blackbody optical fiber thermometers in high temperature environments, J. Thermophys. Heat Transfer 16 (3) (2002) 306312.

[23] ANSYS $^{(2)}$ Academic Research, Release 14.5.0

[24] Rahmatullah, S.T. Mohyud-Din, Variation of parameters method for nonlinear diffusion equations, Int. J. Mod. Appl. Phys. 3 (1) (2013) 48-56.

[25] J. Keckic, Additions to Kamkes treatise VII: variation of parameters for nonlinear second order differential equations, Publikacije Elektrotehničkog fakulteta - Serija Matematika i fizika 544 (1976) 31-36.

[26] M.A. Noor, S.T. Mohyud-Din, A. Waheed, Variation of parameters method for solving fifth-order boundary value problems, Appl. Math. Inf. Sci. 2 (2) (2008) 135-141.

[27] M.P. Bogdanova, On a generalization of the method of variation of parameters Doklady Akademii Nauk BSSR 6 (1962) 285-287.

[28] T.J. Moore, Application of reduced order modeling methods to increase the efficiency of inverse radiative heat transfer problems (Ph.D. dissertation), Brigham Young University, Provo, UT, 2014.

[29] J.A. Wiebelt, Engineering Radiation Heat Transfer, Rinehart and Winston Inc., Holt, 1966.

[30] P. Talukdar, S.C. Mishra, Analysis of conduction-radiation problem in absorbing, emitting and anisotropically scattering media using the collapsed dimension method, Int. J. Heat Mass Transfer 45 (10) (2002) 2159-2168. 